PROCEEDINGS OF THE

AMERICAN MATHEMATICAL SOCIETY

Volume 130, Number 1, Pages 95-102

S 0002-9939(01)06302-X

Article electronically published on July 25, 2001

\title{
COMPOSITION OF INNER MAPPINGS ON THE BALL
}

\author{
JÖRG ESCHMEIER AND ROLAND WOLFF
}

(Communicated by Steven R. Bell)

\begin{abstract}
Suppose that $F: B_{k} \rightarrow B_{m}$ is an inner map and that $G \in$ $H^{\infty}\left(B_{m}\right)^{n}$. We show that the identity

$$
(G \circ F)^{*}=r(G) \circ F^{*}
$$

holds with an abstract boundary value $r(G)$. If the natural compatibility condition $\sigma_{k}^{F^{*}} \ll \sigma_{m}$ is satisfied, then $r(G)=G^{*}$. Here, $\sigma_{k}^{F^{*}}$ denotes the image of the surface measure on $S_{k}$ under $F^{*}$. In particular, $G \circ F$ is inner if $F$ and $G$ are inner and $\sigma_{k}^{F^{*}} \ll \sigma_{m}$. Furthermore, we characterize the boundedness of composition operators on Hardy spaces in terms of the absolute continuity of $\sigma_{k}^{F^{*}}$.
\end{abstract}

\section{INTRODUCTION}

Let $B_{m}$ denote the open unit ball in $\mathbb{C}^{m}, S_{m}=\partial B_{m}$ the unit sphere and $\sigma_{m}$ the normalized surface measure on $S_{m}$. If $h \in H^{1}\left(B_{m}\right)$, then $h^{*}$ denotes its boundary function. As usual, we identify the Hardy space $H^{p}\left(B_{m}\right)$ with the space $H^{p}\left(S_{m}\right)$ of boundary values. If $n \in \mathbb{N}$, then $H^{p}\left(B_{m}\right)^{n}$ denotes the $n$-fold cartesian product of $H^{p}\left(B_{m}\right)$.

Definition 1.1. A holomorphic map $F: B_{k} \rightarrow B_{m}$ is called inner if $\left|F^{*}\right|=1$ holds $\sigma_{k}$-almost everywhere.

The question to be studied in this paper is the following: Suppose that the maps $F: B_{k} \rightarrow B_{m}$ and $G: B_{m} \rightarrow B_{n}$ are inner.

\section{Is $G \circ F$ an inner map?}

If $F$ is an inner function (i.e., $m=1$ ), then it is well known that the composition $G \circ F$ is inner; cf. 9], Theorem 17.5. But if $m \geq 2$, then the situation is different: There are inner maps $F, G$ such that $G \circ F$ is not inner; cf. [9], Remark 17.6. If $F$ is measure preserving in the sense that the Borel measure $\sigma_{k}^{F^{*}}$ on $S_{m}$ defined by

$$
\sigma_{k}^{F^{*}}(A)=\sigma_{k}\left(\left[F^{*}\right]^{-1}(A)\right)
$$

satisfies $\sigma_{k}^{F^{*}}=\sigma_{m}$, then it was shown in [5] that $G \circ F$ is inner. This solves Problem 5 posed by Walter Rudin in [9]. It was pointed out by the referee of this paper that another solution of Rudin's original problem is contained in [4].

Received by the editors May 24, 2000.

2000 Mathematics Subject Classification. Primary 32H02, $46 \mathrm{E} 15$.

Key words and phrases. Inner mapping, composition operator, Henkin measure, abstract boundary values. 
First we demonstrate that the use of composition operators allows a more transparent proof of the result from [5], and at the same time we extend this result to a more general setting: Suppose that $1 \leq p<\infty$. If $h \in L^{p}\left(\sigma_{m}\right)$, then it is easy to check that the composition $h \circ F^{*}$ is well defined (i.e., does not depend on the choice of representatives of $h$ and $F^{*}$ ) if and only if $\sigma_{k}^{F^{*}} \ll \sigma_{m}$ holds. If we assume the last condition, then

$$
\begin{array}{ll}
C_{F}: H^{p}\left(B_{m}\right) \rightarrow H^{p}\left(B_{k}\right), & C_{F} h=h \circ F, \\
C_{F^{*}}: H^{p}\left(S_{m}\right) \rightarrow H^{p}\left(S_{k}\right), & C_{F^{*}} h=h \circ F^{*}
\end{array}
$$

are densely defined closed linear operators such that

$$
\left(C_{F} h\right)^{*}=h \circ F^{*}=C_{F^{*}} h
$$

holds for all $h$ in the ball algebra

$$
A\left(B_{m}\right)=C\left(\overline{B_{m}}\right) \cap \mathcal{O}\left(B_{m}\right) .
$$

Because $A\left(B_{m}\right) \subseteq H^{p}\left(B_{m}\right)$ is dense, $C_{F}$ is bounded if and only if $C_{F^{*}}$ is bounded. In general, composition operators are not bounded (cf. [3]), but if $F$ is measure preserving, then $C_{F^{*}}$ is bounded (even isometric).

Theorem 1.2. Suppose that $F: B_{k} \rightarrow B_{m}$ is an inner map such that $\sigma_{k}^{F^{*}} \ll \sigma_{m}$ and $C_{F}$ is bounded on $H^{p}\left(B_{m}\right), 1 \leq p<\infty$. Then

$$
(G \circ F)^{*}=G^{*} \circ F^{*}
$$

holds for all $G \in H^{p}\left(B_{m}\right)^{n}$.

Proof. If we choose a sequence $\left(h_{i}\right)$ in $A\left(B_{m}\right)^{n}$ with $h_{i} \rightarrow G$ in $H^{p}\left(B_{m}\right)^{n}$, then $G^{*} \circ F^{*}=C_{F^{*}} G^{*}=\lim _{i} C_{F^{*}} h_{i}=\lim _{i}\left(C_{F} h_{i}\right)^{*}=\left(C_{F} G\right)^{*}=(G \circ F)^{*}$.

It should be remarked that the assumption $\sigma_{k}^{F^{*}} \ll \sigma_{m}$ in Theorem 1.2 is superfluous because it is automatically satisfied if $C_{F}$ is bounded. For completeness we have included a discussion of bounded composition operators in the third section of this paper.

If we replace the Euclidian unit balls in Theorem 1.2 by bounded strictly pseudoconvex domains with smooth boundaries and inner maps by holomorphic maps $F: \Omega_{1} \rightarrow \Omega_{2}$ such that $F^{*}\left(\partial \Omega_{1}\right) \subseteq \partial \Omega_{2}$, then the same proof can be used to obtain a corresponding result. We refer to [10], 11] for the definition and the relevant properties of Hardy spaces over smoothly bounded domains.

In this paper, we present an operator theoretic approach to the composition problem for inner maps. It turns out that the necessary compatibility condition $\sigma_{k}^{F^{*}} \ll \sigma_{m}$ is even sufficient for the identity $(G \circ F)^{*}=G^{*} \circ F^{*}$ to hold for bounded holomorphic maps $G$. No further assumptions are required. If the compatibility condition is omitted, then this identity remains valid with $G^{*}$ replaced by an abstract boundary value $r(G)$. We refer to the following section for the definition of the boundary map $r$.

Theorem 1.3. Suppose that $F: B_{k} \rightarrow B_{m}$ is an inner map.

1. The identity

$$
(G \circ F)^{*}=r(G) \circ F^{*}
$$

holds for all $G \in H^{\infty}\left(B_{m}\right)^{n}$. 
2. If $\sigma_{k}^{F^{*}} \ll \sigma_{m}$, then

$$
(G \circ F)^{*}=G^{*} \circ F^{*}
$$

holds for all $G \in H^{\infty}\left(B_{m}\right)^{n}$.

If in the situation of Theorem 1.3 the map $G$ is inner, then clearly (1.2) implies that $G \circ F$ is inner. In the case $m=1$ the condition $\sigma_{k}^{F^{*}} \ll \sigma_{m}$ is always satisfied and, if in addition $F(0)=0$, then $F$ is measure preserving. This can be proved for instance by using the integral formula [9], Remark 1.4. An abstract argument for the first statement is given in the discussion of Henkin measures in the proof of Theorem 1.3 below. It follows from 9], Proposition 17.3, and the closed graph theorem that the composition operator $C_{F}$ is bounded, as well, in the case $m=1$. Thus, Theorems 1.2 and 1.3 are generalizations of [9], Theorem 17.5, to inner maps.

Corollary 1.4. Suppose that $F: B_{k} \rightarrow B_{m}$ and $G: B_{m} \rightarrow B_{n}$ are inner maps.

1. If $\sigma_{k}^{F^{*}} \ll \sigma_{m}$, then $G \circ F$ is inner.

2. If $m=1$, then $G \circ F$ is inner.

The authors would like to thank Xianjun Yin for translating [5].

\section{Proof of Theorem 1.3}

This section constitutes the proof of Theorem 1.3. We suppose that $F=$ $\left(f_{1}, \ldots, f_{m}\right): B_{k} \rightarrow B_{m}$ is an inner map. For brevity, we set in the sequel $\mu=\sigma_{k}^{F^{*}}$ and consider $\mu$ as a Borel measure on $S_{m}$. By the definition of $\mu$, the composition $h \circ F^{*}$ is a well-defined element of $L^{\infty}\left(\sigma_{k}\right)$ for every $h \in L^{\infty}(\mu)$ and therefore induces a bounded multiplication operator $M_{h \circ F^{*}}$ on $L^{2}\left(\sigma_{k}\right)$. We recall that the space $L(H)$ of all bounded linear operators on a Hilbert space $H$ carries a weak*topology from the duality with the space of all trace class operators. In the sequel we make use of the fact that a linear map $X^{*} \rightarrow L(H)$, where $X^{*}$ is the dual of a separable Banach space $X$, is weak*-continuous if and only if it is weak*-WOT sequentially continuous; cf. [2], Proposition I.2.7.

Lemma 2.1. The mapping

$$
L^{\infty}(\mu) \rightarrow L\left(L^{2}\left(\sigma_{k}\right)\right), \quad h \mapsto M_{h \circ F^{*}}
$$

is a weak ${ }^{*}$-continuous $C^{*}$-algebra homomorphism such that

$$
1 \mapsto I \quad \text { and } \quad z_{j} \mapsto M_{f_{j}^{*}} \text { for } 1 \leq j \leq m .
$$

Proof. We only have to prove the continuity of the mapping under consideration and this follows easily if we show that the operator

$$
S: L^{\infty}(\mu) \rightarrow L^{\infty}\left(\sigma_{k}\right), \quad S h=h \circ F^{*}
$$

is weak*-continuous. Let $T: L^{1}\left(\sigma_{k}\right) \rightarrow L^{1}(\mu)$ be the expectation operator as constructed in [2], Proposition VII.2.8. Thus, $T$ is a bounded linear operator such that

$$
\int_{S_{m}}(T \psi) h d \mu=\int_{S_{k}} \psi\left(h \circ F^{*}\right) d \sigma_{k} \quad \text { for all } \psi \in L^{1}\left(\sigma_{k}\right), h \in L^{\infty}(\mu) .
$$

This identity shows that $S=T^{*}$ is the Banach space adjoint of $T$ and in particular $T^{*}$ is weak*-continuous. 
Let us equip the subspace $H^{\infty}\left(B_{m}\right) \subseteq L^{\infty}\left(B_{m}\right)$ (formed with respect to the $2 m$-dimensional Lebesgue measure on $B_{m}$ ) with the relative topology of the canonical weak*-topology of $L^{\infty}\left(B_{m}\right)$. Because $H^{\infty}\left(B_{m}\right)$ is a weak*-closed subspace of $L^{\infty}\left(B_{m}\right)$, it follows from general duality theory that $H^{\infty}\left(B_{m}\right)$ is the dual of the separable Banach space $Q=L^{1}\left(B_{m}\right){ }^{\perp} H^{\infty}\left(B_{m}\right)$. Moreover, the relative weak*topology on $H^{\infty}\left(B_{m}\right)$, as introduced above, coincides with the weak*-topology from the duality with $Q$. It is well known that $\mathbb{C}[z]$ is weak ${ }^{*}$-dense in $H^{\infty}\left(B_{m}\right)$ and that a sequence in $H^{\infty}\left(B_{m}\right)$ is a weak*-zero sequence if and only if it is bounded and converges to 0 pointwise. Furthermore, the boundary mapping $H^{\infty}\left(B_{m}\right) \rightarrow L^{\infty}\left(\sigma_{m}\right)$, $h \mapsto h^{*}$, is a weak ${ }^{*}$-continuous algebra homomorphism.

A Borel measure $\lambda$ on $S_{m}$ is called a Henkin measure if

$$
\int_{S_{m}} h_{i} d \lambda \stackrel{i \rightarrow \infty}{\longrightarrow} 0
$$

holds for every sequence $\left(h_{i}\right)$ in $A\left(B_{m}\right)$ such that $\left(\left.h_{i}\right|_{B_{m}}\right)$ is a weak*-zero sequence in $H^{\infty}\left(B_{m}\right)$. If $m=1$, then it is well known that $\lambda$ is a Henkin measure if and only if $\lambda$ is absolutely continuous with respect to $\sigma_{1}$; cf. Chapter 9 in [8].

Lemma 2.2. The measure $\mu=\sigma_{k}^{F^{*}}$ is a Henkin measure on $S_{m}$.

Proof. If $\left(h_{i}\right)$ is a sequence in $A\left(B_{m}\right)$ such that $\left(\left.h_{i}\right|_{B_{m}}\right)$ is a weak*-zero sequence in $H^{\infty}\left(B_{m}\right)$, then $\left(h_{i} \circ F\right)$ is a weak ${ }^{*}$-zero sequence in $H^{\infty}\left(B_{k}\right)$ and therefore $\left(h_{i} \circ F\right)^{*}=h_{i} \circ F^{*} \stackrel{\text { weak }^{*}}{\longrightarrow} 0$ in $L^{\infty}\left(\sigma_{k}\right)$. This yields

$$
\int_{S_{m}} h_{i} d \mu=\int_{S_{k}} h_{i} \circ F^{*} d \sigma_{k} \stackrel{i \rightarrow \infty}{\longrightarrow} 0 .
$$

As a Henkin measure, $\mu$ is a closed measure in the sense of [1], Section XI.4. More precisely, by Henkin's theorem (Theorem 9.3.1 in [8]) it follows that w*- $\lim _{i}\left(\left.h_{i}\right|_{S_{m}}\right.$ ) $=0$ in $L^{\infty}(\mu)=L^{1}(\mu)^{*}$ for any sequence $\left(h_{i}\right)$ in $A\left(B_{m}\right)$ with the property that $\mathrm{w}^{*}-\lim _{i}\left(\left.h_{i}\right|_{B_{m}}\right)=0$ in $H^{\infty}\left(B_{m}\right)$. Since the closed unit ball of $L^{\infty}(\mu)$ is sequentially weak*-compact, one obtains a well-defined linear mapping $r: H^{\infty}\left(B_{m}\right) \rightarrow L^{\infty}(\mu)$ by setting $r(h)=g$ if there is a sequence $\left(h_{i}\right)$ in $A\left(B_{m}\right)$ with $\mathrm{w}^{*}-\lim _{i}\left(\left.h_{i}\right|_{B_{m}}\right)=h$ in $H^{\infty}\left(B_{m}\right)$ and $\mathrm{w}^{*}-\lim _{i}\left(\left.h_{i}\right|_{S_{m}}\right)=g$ in $L^{\infty}(\mu)$. It is an elementary exercise to show that $r: H^{\infty}\left(B_{m}\right) \rightarrow L^{\infty}(\mu)$ is the unique weak*-continuous algebra homomorphism with

$$
r(h)=\left.h\right|_{S_{m}} \quad \text { for all } h \in A\left(B_{m}\right) .
$$

For the proof of the second part of Theorem [1.3 we need one more elementary observation. If $\mu \ll \sigma_{m}$, then the natural map $L^{\infty}\left(\sigma_{m}\right) \rightarrow L^{\infty}(\mu)$ is a well-defined $\mathrm{C}^{*}$-algebra homomorphism.

Lemma 2.3. If $\mu \ll \sigma_{m}$, then the inclusion map $L^{\infty}\left(\sigma_{m}\right) \rightarrow L^{\infty}(\mu), h \mapsto h$ is weak ${ }^{*}$-continuous.

Proof. Let $h$ be the Radon-Nikodym derivative of $\mu$ with respect to $\sigma_{m}$. Then the operator of multiplication with $h$

$$
L^{1}(\mu) \rightarrow L^{1}\left(\sigma_{m}\right), \quad \psi \mapsto h \psi
$$

is well defined, isometric and its Banach space adjoint is the mapping under consideration. 
Proof of Theorem 1.3. Let $F: B_{k} \rightarrow B_{m}$ be an inner map. It follows from Lemma 2.1 and Lemma 2.2 that the mapping

$$
\Phi: H^{\infty}\left(B_{m}\right) \rightarrow L\left(L^{2}\left(\sigma_{k}\right)\right), \Phi(h)=M_{r(h) \circ F^{*}}
$$

is an $H^{\infty}$-functional calculus of $M_{F^{*}}$. By this we mean a weak*-continuous algebra homomorphism such that (2.1) holds. On the other hand, it is easy to see that the mapping

$$
\Psi: H^{\infty}\left(B_{m}\right) \rightarrow L\left(L^{2}\left(\sigma_{k}\right)\right), \Psi(h)=M_{(h \circ F)^{*}}
$$

is an $H^{\infty}$-functional calculus of $M_{F^{*}}$ in the same sense; cf. the proof of Lemma 2.2. But this functional calculus is unique, because the holomorphic polynomials are weak ${ }^{*}$-dense in $H^{\infty}\left(B_{m}\right)$, and therefore $\Phi=\Psi$. If $G \in H^{\infty}\left(B_{m}\right)^{n}$, then the equality of $\Phi$ and $\Psi$ yields that

$$
(G \circ F)^{*}=r(G) \circ F^{*},
$$

and the first part of Theorem 1.3 is proved.

In order to prove the second part of Theorem 1.3 let us assume that $\mu=\sigma_{k}^{F^{*}} \ll$ $\sigma_{m}$ holds. Since the boundary mapping $H^{\infty}\left(B_{m}\right) \rightarrow L^{\infty}\left(\sigma_{m}\right), h \mapsto h^{*}$, is weak*continuous, Lemma 2.3 yields

$$
r(h)=h^{*} \in L^{\infty}\left(\sigma_{m}\right) \subseteq L^{\infty}(\mu)
$$

for all $h \in H^{\infty}\left(B_{m}\right)$. Together with (1.1) this settles (1.2) and the proof of Theorem 1.3 is complete.

\section{BOUNDED COMPOSITION OPERATORS}

In this section we study the connection between the boundedness of a composition operator $C_{F}$ and the absolute continuity of the image measure $\mu=\sigma_{k}^{F^{*}}$. Let $F: B_{k} \rightarrow B_{m}$ be a holomorphic map (not necessarily inner) and consider $F^{*}$ as a measurable map $F^{*}: S_{k} \rightarrow \overline{B_{m}}$. We denote by $\mu=\sigma_{k}^{F^{*}}$ the Borel measure on $\overline{B_{m}}$ defined by

$$
\mu(A)=\sigma_{k}\left(\left[F^{*}\right]^{-1}(A)\right) .
$$

Lemma 3.1. Let $a, b \in \mathbb{R}, 0<a \leq b$. Suppose that $\varphi: S_{m} \rightarrow \mathbb{R}$ is a Borel measurable function with $a \leq \varphi \leq b$. If $\nu$ is a finite positive Borel measure on $S_{m}$ and if $\delta>0$, then there is a function $f \in A\left(B_{m}\right)$ with $|f| \leq b$ and

$$
\nu\left(\left\{z \in S_{m}: \varphi(z) \neq|f(z)|\right\}\right)<\delta .
$$

A proof of Lemma 3.1 can be found in [6], Lemma 1.6.

Theorem 3.2. Let $1 \leq p<\infty$. If $F: B_{k} \rightarrow B_{m}$ is a holomorphic map such that $C_{F}: H^{p}\left(B_{m}\right) \rightarrow H^{p}\left(B_{k}\right), C_{F} h=h \circ F$, is bounded, then

$$
\mu(A)=\sigma_{k}^{F^{*}}(A) \leq\left\|C_{F}\right\|^{p} \sigma_{m}(A)
$$

holds for all Borel subsets $A$ of $S_{m}$.

Proof. Let $A \subseteq S_{m}$ be a measurable subset and let $0<\varepsilon<1$. We define a measurable function $\varphi: S_{m} \rightarrow \mathbb{R}$ with $\varepsilon \leq \varphi \leq 1$ by

$$
\varphi=\chi_{A}+\varepsilon \chi_{S_{m} \backslash A} .
$$


Let $\delta>0$. By Lemma 3.1 we can find a function $f \in A\left(B_{m}\right)$ with $|f| \leq 1$ and $\left(\sigma_{m}+\mu\right)\left(\left\{z \in S_{m}: \varphi(z) \neq|f(z)|\right\}\right)<\delta$. We then estimate

$$
\begin{aligned}
\int_{S_{m}} \varphi^{p} d \mu & \leq \mu(\{\varphi \neq|f|\})+\int_{S_{m}}|f|^{p} d \mu \\
& \leq \delta+\int_{S_{k}}\left|f \circ F^{*}\right|^{p} d \sigma_{k}=\delta+\left\|C_{F} f\right\|_{H^{p}\left(B_{k}\right)}^{p} \\
& \leq \delta+\left\|C_{F}\right\|^{p} \int_{S_{m}}|f|^{p} d \sigma_{m} \\
& =\delta+\left\|C_{F}\right\|^{p}\left(\int_{A}+\int_{\left(S_{m} \backslash A\right) \cap\{\varphi \neq|f|\}}+\int_{\left(S_{m} \backslash A\right) \cap\{\varphi=|f|\}}\right)|f|^{p} d \sigma_{m} \\
& \leq \delta+\left\|C_{F}\right\|^{p}\left(\sigma_{m}(A)+\sigma_{m}(\{\varphi \neq|f|\})+\varepsilon^{p}\right) \\
& \leq \delta+\left\|C_{F}\right\|^{p}\left(\sigma_{m}(A)+\delta+\varepsilon^{p}\right) .
\end{aligned}
$$

Taking the limit $\delta \downarrow 0$ yields

$$
\mu(A)+\varepsilon^{p} \mu\left(S_{m} \backslash A\right)=\int_{S_{m}} \varphi^{p} d \mu \leq\left\|C_{F}\right\|^{p}\left(\sigma_{m}(A)+\varepsilon^{p}\right),
$$

and the proof of Theorem 3.2 is completed by taking the limit $\varepsilon \downarrow 0$.

Theorem 3.2 allows us to prove the announced characterization of bounded composition operators.

Corollary 3.3. Let $1 \leq p<\infty$ and suppose that $F: B_{k} \rightarrow B_{m}$ is a holomorphic map.

1. If $C_{F}$ is bounded on $H^{p}\left(B_{m}\right)$, then $\left.\mu\right|_{S_{m}} \ll \sigma_{m}$ and its Radon-Nikodym derivative $h=d\left(\left.\mu\right|_{S_{m}}\right) / d \sigma_{m}$ is essentially bounded with

$$
\|h\|_{\infty} \leq\left\|C_{F}\right\|^{p} .
$$

2. If $F$ is an inner map, then the composition operator $C_{F}$ is bounded on $H^{p}\left(B_{m}\right)$ if and only if $\mu \ll \sigma_{m}$ and $h=d \mu / d \sigma_{m}$ is essentially bounded. In this case, equality holds in (3.2).

Proof. If $C_{F}$ is bounded, then $\left.\mu\right|_{S_{m}} \ll \sigma_{m}$ by Theorem 3.2 Let $h$ be the RadonNikodym derivative of $\left.\mu\right|_{S_{m}}$ relative to $\sigma_{m}$. Thus, $h$ is a real-valued, non-negative and integrable function that satisfies

$$
\int_{A} h d \sigma_{m}=\mu(A) \leq\left\|C_{F}\right\|^{p} \sigma_{m}(A)
$$

for all Borel subsets $A \subseteq S_{m}$. Then standard measure theory (Theorem X.5 in [7]) implies that $h$ is essentially bounded with $\|h\|_{\infty} \leq\left\|C_{F}\right\|^{p}$. This proves the first part of Corollary 3.3. In the setting of the second part, the converse follows from the estimate

$$
\begin{aligned}
\left\|C_{F} f\right\|_{H^{p}\left(B_{k}\right)}^{p}=\int_{S_{k}}\left|f \circ F^{*}\right|^{p} d \sigma_{k}=\int_{S_{m}}|f|^{p} d \mu & \\
& =\int_{S_{m}}|f|^{p} h d \sigma_{m} \leq\|h\|_{\infty}\|f\|_{H^{p}\left(B_{m}\right)}^{p},
\end{aligned}
$$

which is valid for all $f \in A\left(B_{m}\right)$. 
In the case of equal dimensions, that is, $k=m$, it is well known that the boundedness of $C_{F}$ implies the absolute continuity of $\sigma_{k}^{F^{*}}$, cf. Corollary 3.38 in 3]. The methods presented in this section will work in a more general situation, as well. The balls $B_{k}$ and $B_{m}$ can be replaced by bounded domains $\Omega_{1} \subset \mathbb{C}^{k}$ and $\Omega_{2} \subset \mathbb{C}^{m}$ with $C^{2}$-boundaries $b \Omega_{1}$ and $b \Omega_{2}$. It is enough to assume that $\Omega_{2}$ is strictly pseudoconvex, in order to ensure the validity of Lemma 3.1. In this context, an inner map $F: B_{k} \rightarrow B_{m}$ has to be replaced by a holomorphic map $F: \Omega_{1} \rightarrow \Omega_{2}$ with $F^{*}(z) \in b \Omega_{2}$ for almost all $z \in b \Omega_{1}$.

\section{REMARKS}

Although we shall not use the following observations in the present paper, they might be of independent interest. If $F \in H^{\infty}\left(B_{k}\right)^{m}$, then the measure $\mu=\sigma_{k}^{F^{*}}$ defined on $\mathbb{C}^{m}$ is supported by the essential range of $F^{*}$ which coincides with the Taylor spectrum $\sigma\left(M_{F^{*}}\right)$ of the tuple of multiplication operators $M_{F^{*}}$ with symbol $F^{*}$ on $L^{2}\left(\sigma_{k}\right)$.

Remark 4.1. The measure $\mu=\sigma_{k}^{F^{*}}$ is a scalar-valued spectral measure of the normal $m$-tuple $M_{F^{*}} \in L\left(L^{2}\left(\sigma_{k}\right)\right)^{m}$.

Indeed, if $E$ denotes the operator-valued spectral measure of $M_{F^{*}}$, then we have $E(A)=M_{\chi_{\left[F^{*}\right]^{-1}(A)}}$ for all Borel subsets $A \subseteq \sigma\left(M_{F^{*}}\right)$. Because of

$$
\|E(A) h\|_{2}^{2}=\int_{\left[F^{*}\right]^{-1}(A)}|h|^{2} d \sigma_{k} \quad \text { for all } h \in L^{2}\left(\sigma_{k}\right),
$$

$E$ and $\mu$ are mutually absolutely continuous, that is, $\mu$ is a scalar-valued spectral measure of $M_{F^{*}}$.

In view of Lemma 2.1, the $L^{\infty}(\mu)$-functional calculus of the normal tuple $M_{F^{*}}$ is given by

$$
h\left(M_{F^{*}}\right)=M_{h \circ F^{*}} \quad \text { for all } h \in L^{\infty}(\mu) .
$$

\section{REFERENCES}

1. Urban Cegrell, Capacities in complex analysis, Aspects of Mathematics, vol. E14, Vieweg, Braunschweig, 1988. MR 89m:32001

2. John B. Conway, The theory of subnormal operators, Mathematical surveys and monographs, vol. 36, Amer. Math. Soc., Providence, R.I., 1991. MR 92h:47026

3. Carl C. Cowen and Barbara D. MacCluer, Composition operators on spaces of analytic functions, CRC Press, Boca Raton, FL, 1995. MR 97i:47056

4. Guangfu Cao, Bounded composition operators on Hardy spaces, Acta Math. Sin. 40 (1997), 777-782. MR 99c:47032

5. Xuan Hao Ding and Shun Hua Sun, Composition of inner maps, Chinese Ann. Math. Ser. A 18 (1997), no. 3, 313-316. MR 98i:32032

6. Jörg Eschmeier, Algebras of subnormal operators on the unit ball, J. Operator Theory 42 (1999), 37-76. MR 2000e:47013

7. Serge Lang, Analysis II, Addison-Wesley, Reading, Mass., 1969.

8. Walter Rudin, Function theory in the unit ball of $\mathbb{C}^{n}$, Grundlehren der mathematischen Wissenschaften, vol. 241, Springer-Verlag, New York, 1980. MR 82i:32002

9. - New constructions of functions holomorphic in the unit ball of $\mathbb{C}^{n}$, CBMS Regional Conference Series in Mathematics, no. 63, AMS, Providence, R.I., 1986. MR 87f:32013 
10. Edgar Lee Stout, $H^{p}$-functions on strictly pseudoconvex domains, Amer. J. Math. 98 (1976), 821-852. MR 54:10671

11. Roland Wolff, Spectral theory on Hardy spaces in several complex variables, Dissertation, Westfälische Wilhelms-Universität Universität Münster, Germany, 1996.

Fachrichtung 6.1 Mathematik, Universität des Saarlandes, Postfach 151150, 66041 SaARbrüCKen, Germany

E-mail address: eschmei@math.uni-sb.de

Fachrichtung 6.1 Mathematik, Universität des Saarlandes, Postfach 151150, 66041 SAARbrüCKen, Germany

E-mail address: wolff@math.uni-sb.de 